\title{
Effects of Magnetic History on AC Loss Minimum in BSCCO-2223 Composite Tape
}

\author{
E. Maievskyi*, M. Ciszek and S. Trojanowski \\ W. Trzebiatowski Institute of Low Temperature and Structure Research, Polish Academy of Sciences, \\ Okólna 2, 50422 Wrocław, Poland
}

\begin{abstract}
In the paper we report experimental results of AC magnetization losses in a multi-filamentary BSCCO-2223 superconducting composite tape due to coaxial AC and DC magnetic fields subjected perpendicularly to the plane of the tape. Such superposition of magnetic fields usually leads to some reduction of magnetization losses. A distinguished minimum in the AC loss is observed, at a certain DC bias magnetic field and at a certain fixed magnetic field amplitude. It is shown that the minimum of the AC losses very strongly depends on magnetic history of the investigated tape, which is directly related to the magnetic flux trapping within tape's superconducting filaments region. Measurements were carried out with a sinusoidally varying magnetic fields at amplitudes up to $100 \mathrm{mT}$ and a superimposed DC magnetic field up to $50 \mathrm{mT}$, at frequency range of 21-113 $\mathrm{Hz}$.
\end{abstract}

DOI: $10.12693 /$ APhysPolA.130.527

PACS/topics: 84.71.Mn, 74.25.N-, 74.25.Sv, 74.25.Wx

\section{Introduction}

Nowadays, one of the main fields of superconductors' applications are various devices used to the transportation and energy industry. A lot of them are connected with the presence of $\mathrm{AC}$ currents and $\mathrm{AC} / \mathrm{DC}$ magnetic fields leading to energy dissipation. The knowledge of the energy dissipation mechanisms in superconducting wires and tapes is extremely crucial.

At present, the most highly developed high temperature superconducting (HTS) material for commercial applications are ceramics of chemical formula $(\mathrm{Bi}, \mathrm{Pb})_{2} \mathrm{Sr}_{2} \mathrm{Ca}_{2} \mathrm{Cu}_{3} \mathrm{O}_{10-\delta}$ (BSCCO-2223), of many fine filaments, embedded in silver matrix.

Many research groups were working on a subject of $\mathrm{AC}$ losses caused by external $\mathrm{AC}$ magnetic field when additionally a DC bias field is imposed on it. It was found that the magnitude of the losses, for the fixed value of the amplitude, depends on the static applied field and a clear minimum of the losses occurs. This interesting phenomenon was observed for the first time with low temperature superconductors [1-4]. A theoretical model describing occurrence of the loss minima was given by Clem [5]. In order to explain experimentally observed deviations from the Clem model some modifications were proposed, e.g. including more realistic surface barrier and pinning behavior when applying magnetic fields $[4,6,7]$. Also AC loss minima observed in HTS, bulk and tapes samples, were reported $[8,9]$.

The experimental results reported here are for a commercially available BSCCO-2223/Ag multifilamentary tape, subjected perpendicularly to an external magnetic fields, AC and DC. Due to structure complexity of the

\footnotetext{
*corresponding author; e-mail: E.Maievskyi@int.pan.wroc.pl
}

HTS ceramics theoretical model and numerical calculations describing phenomenon of loss valley are required.

\section{Experimental}

\subsection{Sample}

BSCCO-2223/Ag (type H, Sumitomo Ltd.) multifilamentary composite high temperature superconducting tape was used in our experiments. Some physical parameters of the sample are given in Table I. The length of used sample tapes was equal to $10 \mathrm{~cm}$.

TABLE I

Specification of BSCCO-2223/Ag multifilamentary tape, Sumitomo Ltd., type H.

\begin{tabular}{r|c}
\hline \hline width $[\mathrm{mm}]$ & 4.3 \\
\hline thickness $[\mathrm{mm}]$ & 0.23 \\
\hline filamentary zone width $[\mathrm{mm}]$ & 4.1 \\
\hline filamentary zone thickness $[\mathrm{mm}]$ & 0.192 \\
\hline critical current @77K [A] & 207 \\
\hline number of filaments & 121 \\
\hline filling factor $(\mathrm{Ag} / \mathrm{BSCCO})$ & $1: 1.7$
\end{tabular}

\subsection{AC loss measurement system}

The source of a high homogeneous magnetic field is a race-track coil fed with two kinds of currents, simultaneously coming from two power supplies, DC and AC ones [10]. Such electromagnetic system allows to get effective magnetic field in the form of $B=B_{D C}+b_{0} \sin 2 \pi f$ within one magnet coil. In our electrical circuit we could obtain maximal magnetic field amplitude $b_{0}$ up to $100 \mathrm{mT}$ at frequency $f$ up to about $100 \mathrm{~Hz}$. The DC bias magnetic field was up to $50 \mathrm{mT}$, depending on the AC field amplitude and its frequency. AC magnetization losses were measured by means of a standard lock-in 
technique. The external AC/DC magnetic fields were oriented perpendicular to the plane of the sample tape. The sensitivity of our measurement system was better than which corresponds to energy loss of about $1 \mathrm{~nJ} / \mathrm{m} / \mathrm{Hz}$. Detailed description of the measurement setup is given in [11].

The measurements were carried out as follows: the AC magnetic field amplitude was adjusted to a desired, fixed value. Keeping the fixed amplitude the DC bias field was raised to its maximal value, and then lowered to zero. In order to remove magnetic flux trapped inside the sample, before each new measurement run, the AC magnetic field was raised to its maximal value $(\approx 0.1 \mathrm{~T})$ and then lowered to zero (demagnetization). All measurements were controlled by the NI LabVIEW Program.

\section{Results and discussion}

Experimental results of energy dissipation $Q_{m}$ and imaginary part of the AC susceptibility $\chi^{\prime \prime}$, for the BSCCO-2223 sample tape, are shown in Fig. 1. As it is seen the loss data show some frequency dependence, at lower range of $\mathrm{AC}$ field amplitudes. For these fields range eddy currents in a silver sheath contribute to the losses $\left(\approx b^{2}\right)$, as well as some magnetic flux flow effects. The imaginary part of the AC susceptibility $\chi^{\prime \prime}$ (Fig. 1b) is an important parameter which shows the ratio of dissipated in a superconductor energy to externally applied magnetic field energy, and can be derived from

$$
\chi^{\prime \prime}=\frac{\mu_{0} Q_{v}}{\pi \chi_{0} b_{0}^{2}}
$$

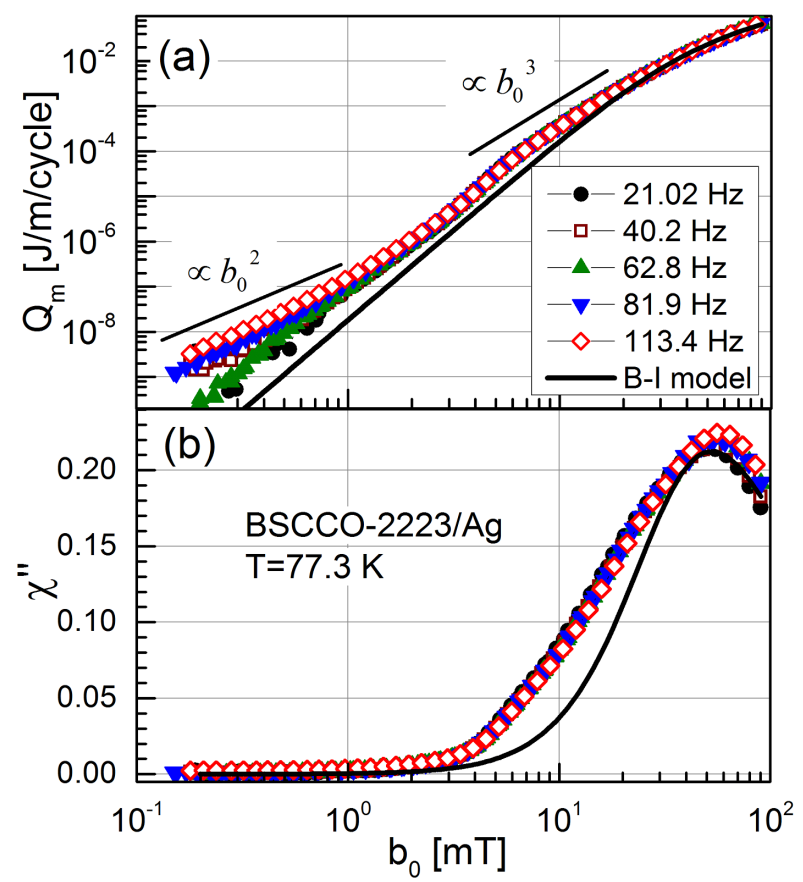

Fig. 1. (a) $\mathrm{AC}$ losses $Q_{m}$ and (b) the imaginary part of the AC susceptibility $\chi^{\prime \prime}$, as a function of the external magnetic field amplitude $b_{0}$. The solid line is according to the Brandt-Indenbom model [13].

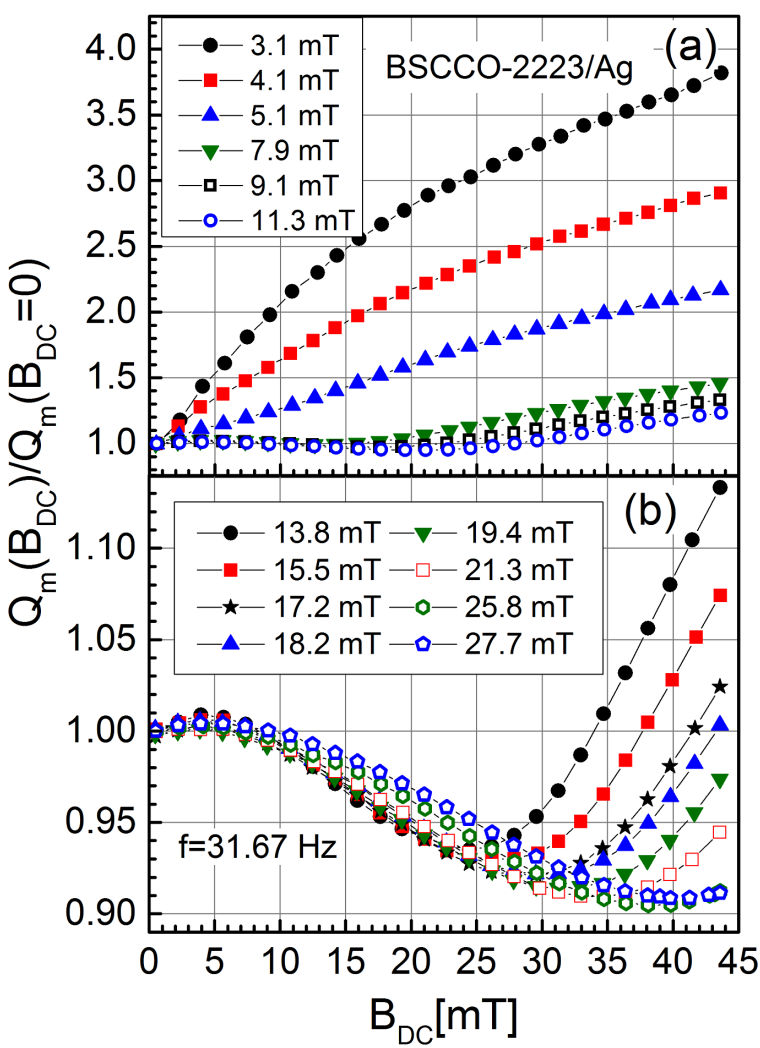

Fig. 2. Normalized magnetization losses in respect to DC bias magnetic field, for different AC magnetic field amplitudes. The loss data is taken for the ascending DC bias field measurements only.

where $Q_{v}$ are the losses per unit volume, $\mu$ is the vacuum permeability, $b$ is an amplitude of external magnetic field, and $\chi$ is demagnetizing factor, related to the effective magnetic field close to a superconductor's body in the Meissner state. The $\chi$ values for different geometrical shapes of superconductors can be found in [12], and for our sample (elliptical cross-section, for dimensions see Table I) equals approximately $\chi \approx 22.4$. The peak in $\chi^{\prime \prime}$, which is related to the full saturation of the sample with magnetic flux, occurs at field amplitude of about $51 \mathrm{mT}$, and this value slightly depends on frequency (changes to $56 \mathrm{mT}$ at $113 \mathrm{~Hz}$ ). The maximal value of $\chi^{\prime \prime}$ does not change very much with the frequency and is equal to about $0.22-0.23$. The solid line represents the theoretical model for AC magnetization losses, in a thin strip, subjected to an external, perpendicular magnetic field, given by Brandt and Indenbom (B-I) [13]. Visible discrepancies, at low amplitudes range, are due to the fact that our tape investigated here differs considerably from B-I model assumptions (a thin strip with a very high aspect ratio). The accuracy of the fitting calculations depends on precision degree of the sample geometrical factors evaluation, such as tape's width and thickness, filamentary zone dimensions, shape of the cross-section, silver to HTS ratio, demagnetizing factor, etc.

Measurements of $\mathrm{AC}$ losses in respect to the $\mathrm{DC}$ bias magnetic field are shown in Fig. 2. For clarity reasons the 
figure is separated in two subfigures ( $a$ and b), each containing data for different range of $\mathrm{AC}$ field amplitudes. The losses are plotted as a function of DC bias field $B_{\mathrm{DC}}$ for different, and fixed, values of the $\mathrm{AC}$ magnetic field amplitudes, $b_{0}$. The data presented here are only for ascending DC field the AC magnetization losses change. For amplitudes smaller than a certain threshold value the losses increase with increasing DC field, whereas for higher amplitudes the losses exhibit a minimum: for example, for $b_{0}=27.7 \mathrm{mT}$ the loss decreases by about $10 \%$ of its initial value at $B_{\mathrm{DC}}=0$. The depth of the minima increases with increasing AC magnetic field amplitudes.

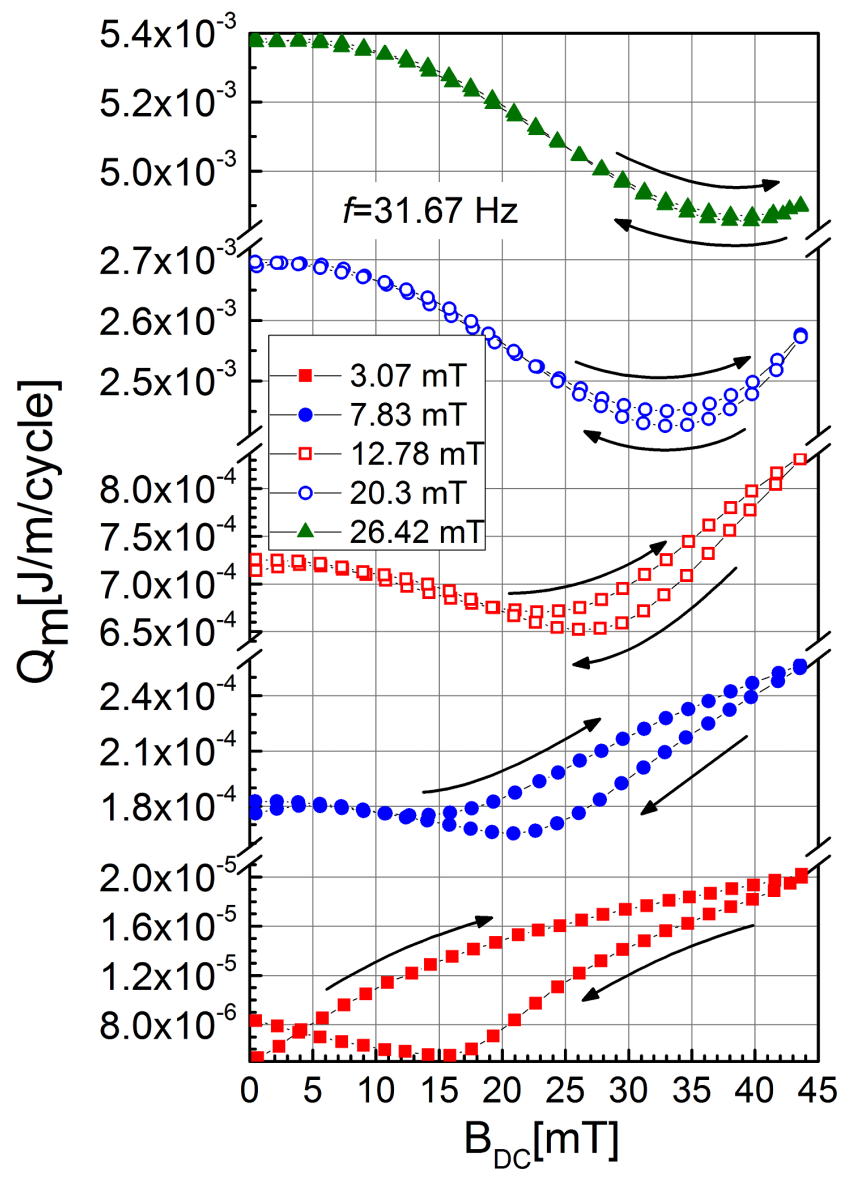

Fig. 3. Energy losses as a function of DC bias magnetic field $B_{\mathrm{DC}}$, for fixed $\mathrm{AC}$ magnetic field amplitudes, $b$. The data are for an ascending and descending DC field (arrows).

Generally, the minimum at losses depends on magnetic history of the sample tape, i.e. different loss characteristics are measured, depending on the DC magnetic field ascending/descending runs (hysteresis). Such typical loss characteristics curves are shown in Fig. 3. For the low amplitudes we observe minima for descending runs of the DC field only, whereas for ascending field runs the losses considerably increases with the DC field. The crossover of the loss curves for ascending and descending DC bias magnetic fields is clear visible for low ampli-

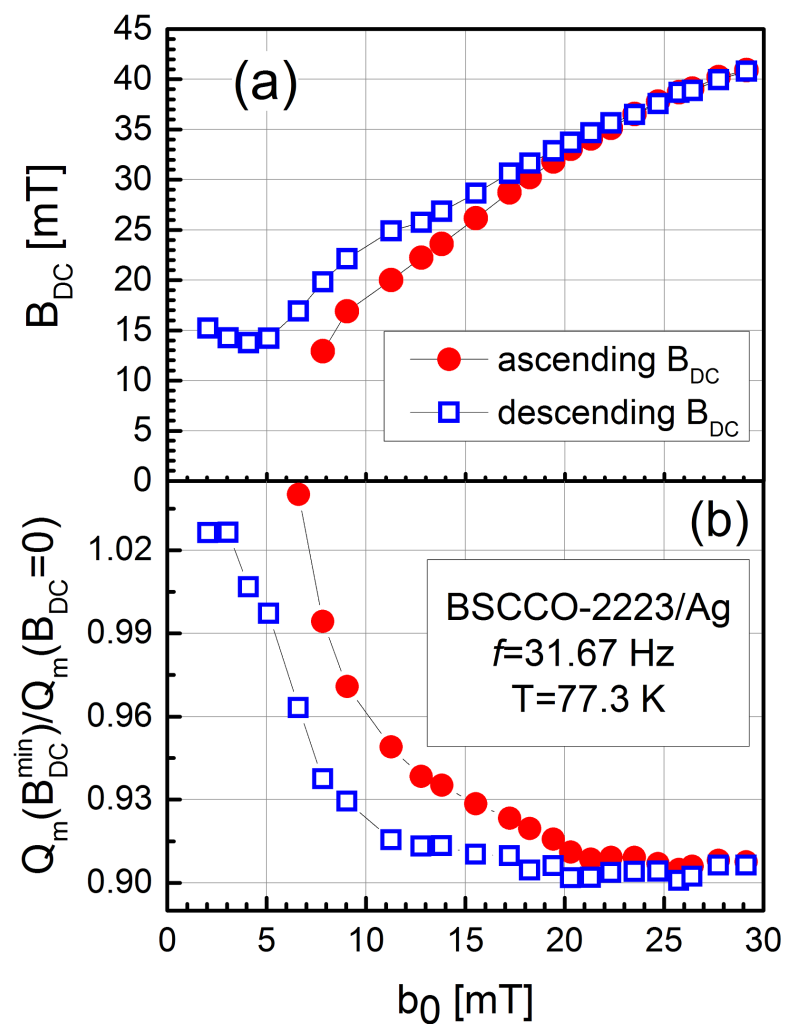

Fig. 4. (a) The DC bias magnetic field $B_{\mathrm{DC}}^{\min }$ at which the minimum in $\mathrm{AC}$ losses occurs as a function of amplitude $b_{0}$. (b) Loss minimum ("valley") depths as a function of $b_{0}$. The data are for ascending and descending DC field runs.

tudes, at about $5 \mathrm{mT}$ of the DC field. The phenomenon of hysteresis is common for low amplitudes only. In turn, for the higher field amplitudes (here, for about 26.4 mT), the hysteresis in losses vanishes.

The hysteresis effects for the wider range of the AC field amplitudes are given in Fig. 4. The upper graph (a) shows the values of the DC bias field at which a minimum occurs, for ascending and descending DC field runs. Both the loss characteristics merge together at amplitudes of about $23 \mathrm{mT}$.

The depths of magnetization loss minima changes due to magnetization history are given in Fig. 4b. The relative change in losses at the minima can fall down to $90 \%$ of its initial value (at $B_{D C}=0$ ). The changes depend on magnetic field amplitudes and magnetic history as well.

Such hysteresis can be explained by presence of a trapped flux in the filamentary core of superconducting tape, causing the actual magnetic induction inside the tape quite different as one can expect taking external magnetic field and any critical state model into consideration.

\section{Conclusions}

In the paper we presented experimental results of AC magnetization losses in commercial multifilamentary BSCCO-2223/Ag tape. The measurements were per- 
formed in coaxially superposed AC and DC bias magnetic field oriented perpendicularly to the flat surface of the sample. For some certain values of the $\mathrm{AC}$ and DC fields clear minima in AC losses characteristics occur. The DC bias magnetic fields at which minima in losses occur are monotonically increasing function of the AC magnetic field amplitude. This phenomenon strongly depends on magnetic history of the sample, i.e. AC loss characteristics are different for ascending and descending DC bias field. This implies that the local magnetic flux distribution within the tape's filaments plays important role. More comprehensive theoretical studying and numerical calculations are required to explain observed in HTS tapes loss minima correctly. This should include more realistic critical state model which takes into considerations critical current density dependence on magnetic field and magnetic flux distribution in a thin HTS strip with a high demagnetizing factor.

\section{References}

[1] J. Thompson, M. Maley, J.R. Clem, J. Appl. Phys. 50, 3531 (1979).

[2] M. Ciszek, G. Kozlowski, P. Tekiel, E.A. Gijsbertse, L.J.M. van de Klundert, Phys. Lett. A 77, 271 (1980).
[3] V.T. Kovachev, J. Appl. Phys. 51, 3812 (1980).

[4] M.A.R. LeBlanc, G. Fillion, J.P. Lorrain, J. Appl. Phys. 59, 3208 (1986).

[5] J.R. Clem, J. Appl. Phys. 50, 3518 (1979).

[6] E.A. Gijsbertse, J. Sikenga, L.J.M. van de Klundert, Physica $B+C$ 106, 59 (1981).

[7] M. Ciszek, P. Tekiel, G. Kozlowski, Supercond. Sci. Technol. 1, 360 (1989).

[8] K.-H. Müller, A.J. Pauza, Phys. C Supercond. 161 , 319 (1989).

[9] S. Celebi, A. Ozturk, U. Kolemen, M.A.R. LeBlanc, J. Appl. Phys. 100, 073912 (2006).

[10] S. Trojanowski, M. Ciszek, E. Maievskyi, Metrol. Meas. Syst. 21, 293 (2014).

[11] E. Maievskyi, M. Ciszek, IEEE Trans. Appl. Superconduct. 25, 8200504 (2015).

[12] P. Fabbricatore, S. Farinon, F. Gömöry, S. Innocenti, Supercond. Sci. Technol. 13, 1327 (2000).

[13] E.H. Brandt, M. Indenbom, Phys. Rev. B 48, 12893 (1993). 